\title{
Analysis of Errors Associated to Observations of Measurement Type
}

\author{
Dhritikesh Chakrabarty \\ Department of Statistics, Handique Girls' College, Guwahati-781001, Assam, India. \\ e-mail:dhritikesh.c@rediffmail.com,dhritikeshchakrabarty@gmail.com
}

\begin{abstract}
Observations or data, collected from an experiment which is free from all sorts of assignable errors suffer from chance error (which is unavoidable or uncontrollable). Consequently the findings obtained by analyzing the observations which are free from the assignable errors are also subject to errors due to the presence of chance error in the observations. Determination of constant(s) associated to mathematical model(s), in different situations, based on the observations is also subject to error due to the same reason. This paper is based on the mathematical model(s) that have been identified for describing the association of chance error(s) in determining constant(s) in some distinct situations where observations/data are of measurement type. Also, chance error has been analyzed in the simplest situation as identified.
\end{abstract}

Key Words: Observation of measurement type; chance error; mathematical model; constant determination.

\section{Introduction}

Observations or data, collected from experiment or survey, normally suffer from various types of errors. Error occurs due to many causes. These causes can be broadly divided into two types namely

1. Assignable cause that is avoidable / controllable

$\& 2$. Chance cause that is unavoidable / uncontrollable.

Even if all the assignable causes of error are controlled or eliminated, observations still do not become free from error. Each of them still suffers from some error which occurs due to some unknown and unintentional cause that is nothing but the chance cause. Findings obtained by analyzing the observations or data which are free from the assignable errors are also subject to errors due to the presence of chance error in the observations. Determination of constant(s), in different 
situations, based on such observations is also subject to error due to the same reason. This leads to the necessity of searching for the association/connection of chance error with observation/data. In the current study, attempt has been made on this aspect of chance error. Mathematical models have been identified for describing the association of chance error in determining constant(s) in some distinct situations where observations/data are of measurement type. Method of determination of constant has been searched for by analyzing chance error in the situation where observations/data consist of itself and chance error.

\section{Gaussian Discovery}

In the year 1809, German mathematician Carl Friedrich Gauss discovered the most significant probability distribution in the theory of statistics popularly known as normal distribution, the credit for which discovery is also given by some authors to a French mathematician Abraham De Moivre who published a paper in 1738 that showed the normal distribution as an approximation to the binomial distribution discovered by James Bernoulli in 1713 [De Moivre, 1711 ; Bernoulli, 1713 ; De Moivre, 1718 ; Walker and Lev, 1965 ; Kendall and Stuart, 1977 \& 1979 ; Stigler, 1982 ; Walker, 1985 ; Brye, 1995 ; Hazewinkel, 2001 ; Marsagilia, 2004 ; Chakrabarty, 2005 \& 2008 ]. The normal probability distribution plays the key role in the theory of statistics as well as in the application of statistics. There are innumerable situations where one can think of applying the theory of normal probability distribution to handle the situations.

The probability density function of normal probability distribution discovered by Gauss is described by the probability density function

$$
\begin{gathered}
f(x: \mu, \sigma)=\left\{\sigma(2 \Pi)^{1 / 2}\right\}^{-1} \cdot \exp \left[-1 / 2\{(x-\mu) / \sigma\}^{2}\right], \\
-\infty<x<\infty,-\infty<\mu<\infty, 0<\sigma<\infty .
\end{gathered}
$$

where (i) $X$ is the associated normal variable,

(ii) $\mu \& \sigma$ are the two parameters of the distribution

and (iii) Mean of $X=\mu$ \& Standard Deviation of $X=\sigma$.

Note: If $\mu=0 \& \sigma=1$,

the density is standardized and $X$ then becomes a standard normal variable.

\subsection{Area Property of Gaussian Distribution}

If $X \sim N(\mu, \sigma)$, then

$$
\text { (i) } P(\mu-1.96 \sigma<X<\mu-1.96 \sigma)=0.95 \text {, }
$$




$$
\begin{aligned}
& \text { (ii) } P(\mu-2.58 \sigma<X<\mu-2.58 \sigma)=0.99 \\
& \text { \& (iii) } P(\mu-3 \sigma<X<\mu-3 \sigma)=0.9973 \text {. }
\end{aligned}
$$

If $X$ is a standard normal variable then

$$
\begin{gathered}
\text { (i) } P(-1.96<X<1.96)=0.95, \\
\text { (ii) } P(-2.58<X<2.58)=0.99 \\
\text { \& (iii) } P(-3<X<3)=0.9973 .
\end{gathered}
$$

\section{Errors in Experimental Observations}

Situations associated to different experiments are different. There are innumerable experiments which can be placed in one of the following situations:

\section{Situation - 1}

In some situations, observation consists of true value of a constant $\mu$ whose value is unknown and to be determined on the basis of observed data.

Let $X_{1}, X_{2}, \ldots \ldots \ldots \ldots, X_{n}$ be $n$ random observations on $\mu$ obtained from an experiment which is free from all sorts of assignable errors. Here, the observations are on $\mu$. Therefore, they should be equal and this common value is nothing but the true value of $\mu$. Since they are different, there exists some cause behind the observations being different. This cause is nothing but the chance cause of error due to which the observations have been compelled to be different. Thus in this situation, each observation $X_{i}$ is composed of true value of $\mu$ and an error $\varepsilon_{i}$.

Thus the observations, in such types of practical situations, satisfy the model

$$
\begin{aligned}
& X_{i}=T(\mu)+\varepsilon_{i}, \quad(i=1,2, \ldots \ldots \ldots, n) \\
& \text { where (i) } X_{i} \text { is the } i^{\text {th }} \text { observation on } \mu, \\
& \text { (ii) } T(\mu) \text { is the true value of } \mu \\
& \text { \& (iii) } \varepsilon_{i} \text { is the error associated to } X_{i} .
\end{aligned}
$$

It is to be noted that the true part of an observation is nothing but the true value of the constant $\mu$ and the observations are collected for determining its true value.

\section{Situation -2}

In some situation, random observations on a pair $(X, Y)$ of two variables $X$ and $Y$ namely

$$
\left(X_{1}, Y_{1}\right),\left(X_{2}, Y_{2}\right), \ldots \ldots \ldots \ldots \ldots \ldots,\left(X_{n}, Y_{n}\right)
$$

are collected to determine the value of an constant $\mu$ which connects the two variables $X$ and $Y$ by the theoretical relationship

$$
f(X)=\mu f(Y) \text { i.e. } \mu=f(X) / \mu f(Y)
$$

for some function $f($.$) .$ 
In this situation, each observation on each of the two variables is influenced by the chance error due to the similar cause mentioned in Situation -1 .

Thus the observations, in such types of practical situations, satisfy the model

$$
\begin{gathered}
X_{i}=T_{i}+\varepsilon_{i} \\
\& Y_{i}=T_{i}^{\prime}+\varepsilon_{i}^{\prime} \\
(i=1,2, \ldots \ldots \ldots . .
\end{gathered}
$$

where (i) $T_{i}$ is the true part of $X_{i}$,

(ii) $\varepsilon_{i}$ is the error associated to $X_{i}$,

(iii) $T_{i}^{\prime}$ is the true part of $Y_{i}$

\& (iv) $\varepsilon_{i}^{\prime}$ is the error associated to $Y_{i}$.

Consequently, for the constant $\mu$ the model becomes

$$
\begin{gathered}
\qquad f\left(T_{i}\right)=\mu f\left(T_{i}^{\prime}\right) \text { i.e. } \mu=f\left(T_{i}\right) / f\left(T_{i}^{\prime}\right) \\
\text { which means } f\left(X_{i}-\varepsilon_{i}\right)=\mu f\left(Y_{i}^{\prime}-\varepsilon_{i}{ }^{\prime}\right) \text { i.e. } \mu=f\left(X_{i}-\varepsilon_{i}\right)^{\prime} f\left(Y_{i}^{\prime}-\varepsilon_{i}{ }^{\prime}\right) \\
(i=1,2, \ldots \ldots \ldots, n) .
\end{gathered}
$$

Thus, the true value of $\mu=f\left(T_{i}\right) / f\left(T_{i}^{\prime}\right)=f\left(X_{i}-\varepsilon_{i}\right) / f\left(Y_{i}^{\prime}-\varepsilon_{i}{ }^{\prime}\right)$.

But, the values of $\mu$ one obtains from the observations are $f\left(X_{i}\right)^{\prime} f\left(Y_{i}\right)(i=1,2, \ldots \ldots \ldots, n)$, which are subject to errors.

\section{Situation -3}

In Situation - 2, it may be so that the constant $\mu$ connects the two variables $X$ and $Y$ by the theoretical relationship

$$
f(X)=\mu g(Y)
$$

for some functions $f($.$) and g($.$) .$

In such types of practical situations, the model will be

$$
f\left(T_{i}\right)=\mu g\left(T_{i}^{\prime}\right) \text { i.e. } \mu=f\left(T_{i}\right) / g\left(T_{i}^{\prime}\right)
$$

which means $f\left(X_{i}-\varepsilon_{i}\right)=\mu g\left(Y_{i}^{\prime}-\varepsilon_{i}^{\prime}\right)$ i.e. $\mu=f\left(X_{i}-\varepsilon_{i}\right) / g\left(Y_{i}^{\prime}-\varepsilon_{i}^{\prime}\right)$

$$
(i=1,2, \ldots \ldots \ldots, n) \text {. }
$$

Thus in this situation,

$$
\text { the true value of } \mu=f\left(T_{i}\right) / g\left(T_{i}^{\prime}\right)=f\left(X_{i}-\varepsilon_{i}\right) / g\left(Y_{i}^{\prime}-\varepsilon_{i}{ }^{\prime}\right) \text {. }
$$

But, the values of $\mu$ one obtains from the observations are $f\left(X_{i}\right) / g\left(Y_{i}\right)(i=1,2$, $n)$, which are subject to errors.

\section{Situation -4}


In Situation - 2, it may be so that the variable $Y$ theoretically depends linearly upon the variable $X$ i.e.

$$
Y=\alpha+\beta X
$$

where $\alpha \& \beta$ are two constants.

In such types of practical situations,

$$
T_{i}^{\prime}=\alpha+\beta T_{i} \quad \text { i.e. } \quad Y_{i}^{\prime}-\varepsilon_{i}^{\prime}=\alpha+\beta .\left(X_{i}-\varepsilon_{i}\right), \quad(i=1,2, \ldots \ldots \ldots, n) .
$$

\section{Situation -5}

In Situation - 2, it may be so that the variable $Y$ theoretically depends quadratically upon the variable $X$ i.e.

$$
Y=\alpha+\beta X+\gamma X^{2}
$$

where $\alpha, \beta \& \gamma$ are three constants.

In such types of practical situations,

$$
T_{i}^{\prime}=\alpha+\beta T_{i}+\gamma T_{i}{ }^{2} \quad \text { i.e. } \quad Y_{i}-\varepsilon_{i}{ }^{\prime}=\alpha+\beta \cdot\left(X_{i}-\varepsilon_{i}\right)+\gamma \cdot\left(X_{i}-\varepsilon_{i}\right)^{2}, \quad(i=1,2, \ldots \ldots \ldots, n) .
$$

\section{Situation - 6 (Generalized Situation of Situation - 4 \& Situation - 5)}

The variable $Y$ theoretically depends upon the variable $X$ by the polynomial of degree $p$ of the form

$$
Y=a_{0}+a_{1} X+a_{2} X^{2}+\ldots \ldots \ldots \ldots+a_{p} X^{p}
$$

where $a_{0}, a_{1}, a_{2}, \ldots \ldots \ldots \ldots, a_{p}$ are constants.

In such types of practical situations, the model will be

$$
T_{i}^{\prime}=a_{0}+a_{1} T_{i}+a_{2} T_{i}^{2}+\ldots \ldots \ldots \ldots+a_{p} T_{i}^{p}
$$

i.e. $Y_{i}-\varepsilon_{i}{ }^{\prime}=a_{0}+a_{1} \cdot\left(X_{i}-\varepsilon_{i}\right)+a_{2} \cdot\left(X_{i}-\varepsilon_{i}\right)^{2}+\ldots \ldots \ldots \ldots . . .+a_{p} .\left(X_{i}-\varepsilon_{i}\right)^{p}$

$$
(i=1,2, \ldots \ldots \ldots, n) \text {. }
$$

\section{Note (Some More Situations)}

Mathematical models have been identified for describing the association of chance error in determining constants in some more distinct situations. Those, along with the above, have been summarized below (Table - I). 
Table - I

Association of Chance Error with the Constant(s) in Different Situations

\begin{tabular}{|c|c|c|c|}
\hline $\begin{array}{c}\text { Serial } \\
\text { No }\end{array}$ & Theoretical relationship & Constant(s) & $\begin{array}{c}\text { Relationship among constants, } \\
\text { observations and errors }\end{array}$ \\
\hline 1. & $f(X)=\mu f(Y)$ & $\mu$ & $f\left(X_{i}-\varepsilon_{i}\right)=\mu f\left(Y_{i}^{\prime}-\varepsilon_{i}^{\prime}\right)$ \\
\hline 2. & $f(X)=\mu g(Y)$ & $\mu$ & $f\left(X_{i}-\varepsilon_{i}\right)=\mu g\left(Y_{i}^{\prime}-\varepsilon_{i}{ }^{\prime}\right)$ \\
\hline 3. & $Y=\alpha+\beta X$ & $\alpha, \beta$ & $Y_{i}-\varepsilon_{i}^{\prime}=\alpha+\beta .\left(X_{i}-\varepsilon_{i}\right)$ \\
\hline 4. & $Y=\alpha+\beta X+\gamma X^{2}$ & $\alpha, \beta, \gamma$ & $Y_{i}-\varepsilon_{i}{ }^{\prime}=\alpha+\beta \cdot\left(X_{i}-\varepsilon_{i}\right)+\gamma \cdot\left(X_{i}-\varepsilon_{i}\right)^{2}$ \\
\hline 5. & $\begin{array}{c}Y=a_{0}+a_{1} X+a_{2} X^{2}+ \\
\ldots \ldots \ldots \ldots+a_{p} X^{p}\end{array}$ & $a_{0}, a_{1}, a_{2}, \ldots ., a_{p}$ & $\begin{array}{c}Y_{i}-\varepsilon_{i}^{\prime}=a_{0}+a_{1} \cdot\left(X_{i}-\varepsilon_{i}\right)+a_{2} \cdot\left(X_{i}-\right. \\
\left.\varepsilon_{i}\right)^{2}+\ldots . .+a_{p}\left(X_{i}-\varepsilon_{i}\right)^{p}\end{array}$ \\
\hline 6. & $f(X, Y)=\mu$ & $\mu$ & $f\left(Y_{i}-\varepsilon_{i}^{\prime}, X_{i}-\varepsilon_{i}\right)=\mu$ \\
\hline 7. & $Y=\lambda \exp (-v X)$ & $\lambda, v$ & $Y_{i}-\varepsilon_{i}^{\prime}=\lambda \exp \left\{-v\left(X_{i}-\varepsilon_{i}\right)\right\}$ \\
\hline 8. & $Y=\lambda \exp \left(-v X^{-1}\right)$ & $\lambda, v$ & $Y_{i}-\varepsilon_{i}^{\prime}=\lambda \exp \left\{-v\left(X_{i}-\varepsilon_{i}\right)^{-1}\right\}$ \\
\hline 9. & $Y=\mu+\lambda \exp (-v X)$ & $\mu, \lambda, v$ & $Y_{i}-\varepsilon_{i}^{\prime}=\mu+\lambda \exp \left\{-v\left(X_{i}-\varepsilon_{i}\right)\right\}$ \\
\hline 10. & $Y=\mu+\lambda \exp \left(-v X^{-1}\right)$ & $\mu, \lambda, v$ & $Y_{i}-\varepsilon_{i}^{\prime}=\mu+\lambda \exp \left\{-v\left(X_{i}-\varepsilon_{i}\right)^{-1}\right\}$ \\
\hline 11. & $Y=\{\alpha+\beta \exp (\gamma X)\}^{-1}$ & $\alpha, \beta, \gamma$ & $Y_{i}-\varepsilon_{i}^{\prime}=\left[\alpha+\beta \exp \left\{\gamma\left(X_{i}-\varepsilon_{i}\right)\right]^{-1}\right.$ \\
\hline 12. & $Y=\mu+\lambda X^{-1}$ & $\mu, \lambda$ & $Y_{i}-\varepsilon_{i}^{\prime}=\mu+\lambda\left(X_{i}-\varepsilon_{i}\right)^{-1}$ \\
\hline 13. & $Y=f\left(X: \mu_{1}, \mu_{2}, \ldots \ldots, \mu_{k}\right)$ & $\mu_{1}, \mu_{2}, \ldots \ldots, \mu_{k}$ & $Y_{i}-\varepsilon_{i}^{\prime}=f\left(X_{i}-\varepsilon_{i}: \mu_{1}, \mu_{2}, \ldots \ldots, \mu_{k}\right)$ \\
\hline 14. & $h(X, Y)=\mu$ & $\mu$ & $h\left(X_{i}-\varepsilon_{i}, Y_{i}-\varepsilon_{i}{ }^{\prime}\right)=\mu$ \\
\hline 15. & $\begin{array}{c}Y=\theta \exp \left\{g(X): \mu_{1}, \mu_{2},\right. \\
\left.\left.\ldots \ldots \ldots, \mu_{k}\right)\right\}\end{array}$ & $\theta, \mu_{1}, \mu_{2}, \ldots \ldots, \mu_{k}$ & $\begin{array}{c}Y_{i}-\varepsilon_{i}{ }^{\prime}=\theta \exp \left\{g\left(X_{i}-\varepsilon_{i}: \mu_{1}, \mu_{2},\right.\right. \\
\left.\left.\ldots \ldots \ldots, \mu_{k}\right)\right\}\end{array}$ \\
\hline
\end{tabular}

In the table, $X$ and $Y$ are two variables where $Y$ theoretically depends upon $X$ and

$$
\left(X_{1}, Y_{1}\right),\left(X_{2}, Y_{2}\right), \ldots \ldots \ldots \ldots \ldots \ldots,\left(X_{n}, Y_{n}\right)
$$

are random observations on the pair $(X, Y)$.

\section{Analysis of errors (Situation - 1)}

Let us consider Situation -1 where observations consist of the true value of a constant $\mu$ whose value is unknown and is to be determined.

In this situation If $X_{1}, X_{2}, \ldots \ldots, X_{n}$ are $n$ random observations on $\mu$, we have

$$
X_{i}=T(\mu)+\varepsilon_{i} \quad, \quad(i=1,2, \ldots \ldots, n)
$$

where (i) $X_{i}$ is the $i^{\text {th }}$ observation on $\mu$,

(ii) $T(\mu)$ is the true value of $\mu$

\& (iii) $\varepsilon_{i}$ is the chance error associated to $X_{i}$. 
Here $\varepsilon_{1}, \varepsilon_{2}, \ldots \ldots, \varepsilon_{n}$ are values of the chance error variable $\varepsilon$ associated to $X_{1}, X_{2}, \ldots \ldots, X_{n}$ respectively.

It is to be noted that

(1) $X_{1}, X_{2}, \ldots \ldots, X_{n}$ are known,

(2) $T(\mu), \varepsilon_{1}, \varepsilon_{2}, \ldots \ldots, \varepsilon_{n}$ are unknown

\& (3) the number of linear equations in (8) is $n$ with $(n+1)$ unknowns implying that the equations are not solvable mathematically.

Reasonable facts /Assumptions regarding $\varepsilon_{i}$ :

(1) $\varepsilon_{1}, \varepsilon_{2}, \ldots \ldots \ldots, \varepsilon_{n}$ are unknown values of the variables $\varepsilon$.

(2) The values $\varepsilon_{1}, \varepsilon_{2}, \ldots \ldots \ldots \ldots, \varepsilon_{n}$ are very small relative to the respective values $X_{1}, X_{2}, \ldots \ldots \ldots \ldots, X_{n}$.

(3) The variable $\varepsilon$ can assume both positive and negative values.

(4) $P(-a-d a<\varepsilon<-a)=P(a<\varepsilon<a+d a)$ for every real $a$.

(5) $P(a<\varepsilon<a+d a)>P(b<\varepsilon<b+d b)$

$\& P(-a-d a<\varepsilon<-a)<P(-b-d b<\varepsilon<-b)$

for every real positive $a<b$.

(6) The facts (3), (4) \& (5) together imply that $\varepsilon$ obeys the normal probability law.

(7) Sum of all possible values of each $\varepsilon$ is 0 (zero) which together with the fact (6) implies that $E(\varepsilon)=0$.

(8) Standard deviation of $\varepsilon$ is unknown and small, say $\sigma_{\varepsilon}$.

(9) The facts (6), (7) \& (8) together imply that $\varepsilon$ obeys the normal probability law with mean (expectation) 0 \& standard deviation $\sigma_{\varepsilon}$. Thus

$$
\varepsilon \sim N\left(0, \sigma_{\varepsilon}\right)
$$

\subsection{Confidence Interval of Error ' $\varepsilon$ '}

Since $\varepsilon \sim N\left(0, \sigma_{\varepsilon}\right)$, the area property of Gaussian distribution given by the equation.5,is

$$
P\left(-1.96 \sigma_{\varepsilon}<\varepsilon<-1.96 \sigma_{\varepsilon}\right)=0.95
$$

i.e. the interval

$$
\left(-1.96 \sigma_{\varepsilon}, 1.96 \sigma_{\varepsilon}\right)
$$

is the $95 \%$ confidence interval of $\varepsilon$.

This means that out of 100 random observations on $\varepsilon$ (unknown), maximum 5 observations fall outside this interval.

Again by the area property of Gaussian distribution given by the equations (6) and (7),

$$
P\left(-2.58 \sigma_{\varepsilon}<\varepsilon<-2.58 \sigma_{\varepsilon}\right)=0.99
$$




$$
\& P\left(-3 \sigma_{\varepsilon}<\varepsilon<3 \sigma_{\varepsilon}\right)=0.9973
$$

respectively which implies that the intervals

$$
\begin{aligned}
& \left(\begin{array}{ll}
-2.58 \sigma_{\varepsilon}, & 2.58 \sigma_{\varepsilon}
\end{array}\right) \\
& \&\left(-3 \sigma_{\varepsilon}, 3 \sigma_{\varepsilon}\right)
\end{aligned}
$$

are respectively the $99 \% \& 99.73 \%$ confidence intervals of $\varepsilon$.

These respectively mean that out of 100 random observations on $\varepsilon$ (unknown), maximum 1 observation falls outside the interval ( $-2.58 \sigma_{\varepsilon}, 2.58 \sigma_{\varepsilon}$ ) and out of 10000 random observations on $\varepsilon$ (unknown), maximum 27 observations fall outside the interval $\left(\begin{array}{ll}-3 \sigma_{\varepsilon} & , 3 \sigma_{\varepsilon}\end{array}\right)$.

\subsection{Confidence Interval of the Parameter ' $\mu$ '}

Also under the assumption numbered (9),

$$
X-\mu \sim N\left(0, \sigma_{\varepsilon}\right) .
$$

This implies that $X \sim N\left(\mu, \sigma_{\varepsilon}\right)$.

Thus by the same area property of Gaussian distribution mentioned above,

$$
\begin{gathered}
P\left(X-1.96 \sigma_{\varepsilon}<\mu<X-1.96 \sigma_{\varepsilon}\right)=0.95, \\
P\left(X-2.58 \sigma_{\varepsilon}<\mu<X-2.58 \sigma_{\varepsilon}\right)=0.99 \\
\& P\left(X-3 \sigma_{\varepsilon}<\mu<X-3 \sigma_{\varepsilon}\right)=0.9973
\end{gathered}
$$

Theses imply the intervals

$$
\begin{aligned}
& \left(X-1.96 \sigma_{\varepsilon}, \quad X-1.96 \sigma_{\varepsilon}\right), \\
& \left(X-2.58 \sigma_{\varepsilon}, \quad X-2.58 \sigma_{\varepsilon}\right) \\
& \&\left(X-3 \sigma_{\varepsilon}, \quad X-3 \sigma_{\varepsilon}\right)
\end{aligned}
$$

are respectively the $95 \%, 99 \%$ and $99.73 \%$ confidence intervals of the parameter $\mu$.

These respectively mean that

(i) out of 100 random intervals corresponding to 100 random observations (or 100 random samples), the value of $\mu$ will fall outside a maximum 5 such intervals defined by the equation (19),

(ii) out of 100 random intervals corresponding to 100 random observations (or 100 random samples), the value of $\mu$ will fall outside a maximum 1 such interval defined by the equation (20)

\& (iii) out of 10000 random intervals corresponding to 10000 random observations (or 10000 random samples), the value of $\mu$ will fall outside a maximum 27 such intervals defined by equation (21). 


\subsection{Confidence Interval of the Observation Variable ' $X$ '}

Again under the assumption numbered (9),

$$
X-\mu \sim N\left(0, \sigma_{\varepsilon}\right) .
$$

This implies that $X \sim N\left(\mu, \sigma_{\varepsilon}\right)$.

Thus by the same area property of Gaussian distribution mentioned above,

$$
\begin{aligned}
& P\left(\mu-1.96 \sigma_{\varepsilon}<X<\mu-1.96 \sigma_{\varepsilon}\right)=0.95 \\
& P\left(\mu-2.58 \sigma_{\varepsilon}<X<\mu-2.58 \sigma_{\varepsilon}\right)=0.99 \\
& \& P\left(\mu-3 \sigma_{\varepsilon}<X<\mu-3 \sigma_{\varepsilon}\right)=0.9973
\end{aligned}
$$

Theses imply the intervals

$$
\begin{aligned}
& \left(\mu-1.96 \sigma_{\varepsilon}, \mu-1.96 \sigma_{\varepsilon}\right), \\
& \left(\mu-2.58 \sigma_{\varepsilon}, \quad \mu-2.58 \sigma_{\varepsilon}\right) \\
\& & \left(\mu-3 \sigma_{\varepsilon} \quad, \quad \mu-3 \sigma_{\varepsilon}\right)
\end{aligned}
$$

are respectively the $95 \%, 99 \%$ and $99.73 \%$ confidence intervals of the variable $X$.

These respectively mean that

(i) out of 100 random observations, maximum 5 observations fall outside the interval given by the equation (25),

(ii) out of 100 random observations, maximum 1 observation falls outside the interval given by equation (26)

\& (iii) out of 10000 random observations, maximum 27 observations fall outside the interval given by equation (27)

\subsection{Least Squares Estimator (LSE) of the Parameter $\mu$}

The LSE $\mu$ is obtained by minimizing

$$
\Sigma \varepsilon_{i}^{2}=\sum_{i=1}^{n}\left(Y_{i}-\mu\right)^{2}
$$

with respect to $\mu$.

The said estimator is thus

$$
\wedge=(1 / n) \underset{\substack{\sum \\ i=1}}{n}
$$

\subsection{Maximum Likelihood Estimator (MLE) of the Parameter $\mu$}

The likelihood function of the observations 


$$
Y_{1}, Y_{2}, \ldots \ldots \ldots, Y_{n}
$$

is given by

$$
L\left(Y_{1}, Y_{2}, \ldots ., Y_{n}: \mu, \sigma\right)=\prod_{i=1}^{n}\left\{\sigma(2 \Pi)^{1 / 2}\right\}^{-1} \cdot \exp \left[-1 / 2\{(x-\mu) / \sigma\}^{2}\right]
$$

which gives

$$
\log _{e} L\left(Y_{1}, Y_{2}, \ldots ., Y_{n}: \mu, \sigma\right)=\sum_{i=1}^{n}\left\{\sigma(2 \Pi)^{1 / 2}\right\}^{-1} \cdot \exp \left[-1 / 2\{(x-\mu) / \sigma\}^{2}\right]
$$

Maximizing this with respect to $\mu$ its MLE is found to be

$$
\hat{\mu}=(1 / n) \sum_{i=1}^{n} Y_{i}
$$

\subsection{Error in Estimator of $\mu$}

Since

$$
Y_{i}=\mu+\varepsilon_{i}
$$

therefore,

$$
\stackrel{\wedge}{\mu}=(1 / n) \underset{i=1}{\sum} Y_{i}=\mu+(1 / n) \underset{i=1}{\sum} \varepsilon_{i}
$$

This means, the estimator (both LSE and MLE) of $\mu$ suffers from an error

$$
(1 / n) \sum_{i=1}^{n} \varepsilon_{i}
$$

which may not be 0 (zero).

\subsection{Estimator of Error ' $\varepsilon$ '}

Since

$$
\begin{aligned}
Y_{i} & =\mu+\varepsilon_{i} \\
\wedge & \wedge \\
\& \quad & =(1 / n) \sum_{i=1}^{n} Y_{i}
\end{aligned}
$$

therefore, the estimator (both LSE and MLE) of error $\varepsilon_{i}$ associated to the observation $Y_{i}$ is

$$
\varepsilon_{i}=\stackrel{\wedge}{Y_{i}}-\mu={ }_{Y_{i}}-(1 / n) \underset{i=1}{\sum} Y_{i}
$$




\section{Analysis of Annual Maximum Temperature at Guwahati}

The annual maximum temperature at a location satisfies the Situation -1 if the change in that is free from assignable cause(s). Assuming that the change in temperature at Guwahati is free from assignable cause, the observations on the annual maximum temperature at Guwahati has been analyzed in order to obtain various estimates possible. Values of annual maximum Temperature at Guwahati observed during the period from 1969 to 2010 have been presented in Table - II. Various estimates, obtained, have been presented in Table - III, Table - IV and Table -V.

Table - II

Observed values of Annual Maximum Temperature at Guwahati (in Degree Celsius)

\begin{tabular}{|c|c|c|c|c|c|c|c|}
\hline Year & $\begin{array}{c}\text { Observed } \\
\text { value }\end{array}$ & Year & $\begin{array}{c}\text { Observed } \\
\text { value }\end{array}$ & Year & $\begin{array}{c}\text { Observed } \\
\text { value }\end{array}$ & Year & $\begin{array}{c}\text { Observed } \\
\text { value }\end{array}$ \\
\hline 1969 & 37.1 & 1979 & 38.6 & 1989 & 36.7 & 2002 & 35.7 \\
\hline 1970 & 36.6 & 1980 & 35.1 & 1990 & 36.0 & 2003 & 37.4 \\
\hline 1971 & 36.0 & 1981 & 35.8 & 1991 & 37.4 & 2004 & 38.0 \\
\hline 1972 & 35.7 & 1982 & 36.5 & 1992 & 39.4 & 2005 & 36.6 \\
\hline 1973 & 39.0 & 1983 & 36.7 & 1993 & 36.4 & 2006 & 38.0 \\
\hline 1974 & 36.1 & 1984 & 37.2 & 1994 & 37.3 & 2007 & 37.3 \\
\hline 1975 & 39.2 & 1985 & 36.5 & 1995 & 36.3 & 2008 & 37.3 \\
\hline 1976 & 39.0 & 1986 & 38.4 & 1996 & 37.2 & 2009 & 38.0 \\
\hline 1977 & 35.3 & 1987 & 37.2 & 2000 & 37.5 & 2010 & 37.2 \\
\hline 1978 & 36.8 & 1988 & 36.3 & 2001 & 36.7 & 2011 & - \\
\hline
\end{tabular}

Table - III

Estimated Value of Annual Maximum Temperature at Guwahati (in Degree Celsius)

\begin{tabular}{|c|l|c|}
\hline Serial No. & \multicolumn{1}{|c|}{ Estimator } & Estimate \\
\hline 1 & Mean & 37.0641 \\
\hline 2 & Standard Deviation & 1.0569 \\
\hline 3 & $95 \%$ Confidence Interval & $(34.99245 \quad 39.13575)$ \\
\hline 4 & $99 \%$ Confidence Interval & $(34.33713 \quad$, 39.79107) \\
\hline 5 & $99.73 \%$ Confidence Interval (Natural Interval) & $(33.89321 \quad$ 40.23499) \\
\hline 6 & $\begin{array}{c}\text { Number of Observations falling out side of the } \\
\text { Corresponding Natural Intervals }\end{array}$ \\
\hline
\end{tabular}


Table - IV

Estimate of Chance Error (in Degree Celsius)

\begin{tabular}{|c|l|c|}
\hline \multirow{2}{*}{ Serial No. } & \multicolumn{1}{|c|}{ Parameter related to Chance Error } & \multicolumn{2}{|c|}{ Estimate } \\
\hline 1 & Mean & \multicolumn{2}{|c|}{1.03241} \\
\hline 2 & Standard Deviation & $(-2.07165 \quad, \quad 2.07165)$ \\
\hline 3 & $95 \%$ Confidence Interval & $(-2.72697 \quad, \quad 2.72697)$ \\
\hline 4 & $99 \%$ Confidence Interval & $(-3.1709 \quad, \quad 3.1709)$ \\
\hline 5 & $99.73 \%$ Confidence Interval (Natural Interval) & 0 \\
\hline 6 & $\begin{array}{c}\text { Number of Observations falling out side of the } \\
\text { Corresponding Natural Intervals }\end{array}$ \\
\hline
\end{tabular}

Table $-\mathrm{V}$

Estimate of Chance Error associated to Observation (in Degree Celsius)

\begin{tabular}{|c|c|c|c|c|c|}
\hline Year & $\begin{array}{c}\text { Annual Maximum } \\
\text { Temperature }\end{array}$ & $\begin{array}{c}\text { Estimate of } \\
\text { Error }\end{array}$ & Year & $\begin{array}{c}\text { Annual Maximum } \\
\text { Temperature }\end{array}$ & $\begin{array}{c}\text { Estimate of } \\
\text { Error }\end{array}$ \\
\hline 1969 & 37.1 & 0.0359 & 1989 & 36.7 & -0.3641 \\
\hline 1970 & 36.6 & -0.4641 & 1990 & 36.0 & -1.0641 \\
\hline 1971 & 36.0 & -1.0641 & 1991 & 37.4 & 0.3359 \\
\hline 1972 & 35.7 & -1.3641 & 1992 & 39.4 & 2.3359 \\
\hline 1973 & 39.0 & 1.9359 & 1993 & 36.4 & -0.6641 \\
\hline 1974 & 36.1 & -0.9641 & 1994 & 37.3 & 0.2359 \\
\hline 1975 & 39.2 & 2.1359 & 1995 & 36.3 & -0.7641 \\
\hline 1976 & 39.0 & 1.9359 & 1996 & 37.2 & 0.1359 \\
\hline 1977 & 35.3 & -1.7641 & 2000 & 37.5 & 0.4359 \\
\hline 1978 & 36.8 & -0.2641 & 2001 & 36.7 & -0.3641 \\
\hline 1979 & 38.6 & 1.5359 & 2002 & 35.7 & -1.3641 \\
\hline 1980 & 35.1 & -1.9641 & 2003 & 37.4 & 0.3359 \\
\hline 1981 & 35.8 & -1.2641 & 2004 & 38.0 & 0.9359 \\
\hline 1982 & 36.5 & -0.5641 & 2005 & 36.6 & -0.4641 \\
\hline 1983 & 36.7 & -0.3641 & 2006 & 38.0 & 0.9359 \\
\hline 1984 & 37.2 & 0.1359 & 2007 & 37.3 & 0.2359 \\
\hline 1985 & 36.5 & -0.5641 & 2008 & 37.3 & 0.2359 \\
\hline 1986 & 38.4 & 1.3359 & 2009 & 38.0 & 0.9359 \\
\hline 1987 & 37.2 & 0.1359 & 2010 & 37.2 & 0.1359 \\
\hline 1988 & 36.3 & -0.7641 & & & \\
\hline
\end{tabular}




\section{Conclusion}

1. In this study, analysis of error has been developed for the situation-1 only. Analysis of error is required to be attempted for each of the other situations, where associations of errors have been identified, discussed in this paper.

2. The situation discussed here corresponds to theoretically known relationships between two variables. There is necessity for studying the associations of errors in the situations where more than two variables are related by known theoretical relationships.

3. There is also necessity for studying the associations of errors in the situations where two and /or more variables are related by unknown theoretical relationships.

\section{References}

Brye W., The Normal Distribution: Characterizations with Applications , published by Springer Verlag, ISBN 0 - 387 - 97990 - 5. 1995.

Bernoulli J., Arts Conjectandi, published by Impensis Thurmisiorum Fratrum Basileae. 1713.

Chakrabarty D., Probabilistic Forecasting of Time Series, Project Report (2002 - 2005), University Grants Commission. 2005.

Chakrabarty D., Probability: Link between the Classical Definition and the Empirical Definition, $J$. Ass. Sc. Soc., 45, 13-18, June 2005.

Chakrabarty D., Bernoulli's Definition of Probability: Special Case of Its Chakrabarty's Definition, Int. J. Agricult. Stat. Sci., 4(1), 23 - 27, 2008.

De Moivre A., De Mensura Sortis, published by Philosophical Transaction of the Royal Society,1711.

De Moivre A., The Doctrine of Chances, $1^{\text {st }}$ Edition (2nd Edition in 1738 \& 3rd Edition in 1756), ISBN $0-8218-2103-2.1718$.

Grant E. L., Statistical Quality Control ”, published by McGraw Hill, 1972.

Hazewinkel M., Normal Distribution, Encyclopedia of Mathematics, Springer, ISBN 978 - 1 $55608-010-4,2001$.

Kendall M. G. and Stuart A., Advanced Theory of Statistics, Vol. 1 \& 2, $4^{\text {th }}$ Edition, New York, Hafner Press, 1977.

Marsagilia G., Evaluating the Normal Distribution, Journal of Statistical Software, 11 (4), 2004.

Shewhart W. A., Economic Control of Quality of Manufactured Product, published by Van Nastrand, 1931. 
Stigler S. M., A Modest Proposal : A New Standard for the Normal, The American Statistician, 36 (2), $137-138,1982$.

Walker H. M. \& Lev J., Statistical Inference, Oxford \& IBH Publishing Company. 1965.

Walker H. M., De Moivre on the Law of Normal Probability, In Smith, David Eugene. A Source Book in Mathematics, Dover, ISBN 0 - 486 - 64690 - 4, 1985. 